\title{
Characteristics of the interface of a laser-quenched steel substrate and chromium electroplate
}

\author{
Huaixue Li ${ }^{\text {a,b,*, }}$, Guangnan Chen ${ }^{a}$, Guoxiang Zhang ${ }^{\text {a,b }}$, Kun Zhang ${ }^{\text {a }}$, Gengxing Luo ${ }^{\text {a }}$ \\ ${ }^{a}$ Surface Modification Laboratory, Institute of Mechanics, Chinese Academy of Sciences, Beijing, 100080, PR China \\ ${ }^{\mathrm{b}}$ Graduate School of the Chinese Academy of Sciences, Beijing, PR China
}

Received 9 June 2005; accepted in revised form 4 January 2006

Available online 21 February 2006

\begin{abstract}
The interface of a laser-discrete-quenched steel substrate and as-deposited chromium electroplate was investigated by ion beam etching, dissolving-substrate-away and using a Vickers microhardness tester, in an attempt to reveal the mechanism that the service life of the chromium-coated parts is increased by the duplex technique of laser pre-quenching plus chromium post-depositing. The laser quenching of the steel substrate can reduce the steep hardness gradient at the substrate/chromium interface and improve the load-bearing capacity of chromium electroplate. Moreover, the laser quenching prior to plating has an extremely great effect on the morphologies and microstructure of the substrate/chromium interface: there is a transient interlayer at the original substrate/chromium interface while there is not at the laser-quenchedzone/chromium interface; the near-substrate surface microstructure and morphologies of the free-standing chromium electrodeposits, whose substrate was dissolved away with nital $30 \%$ in volume, inherit the periodically gradient characteristics of the laser-discrete-quenched substrate surface.
\end{abstract}

(C) 2006 Elsevier B.V. All rights reserved.

Keywords: Interfaces; Electroplating; Chromium; Laser quenching

\section{Introduction}

Electroplated chromium coatings have been extensively used on engineering parts and composites such as piston rings, work rolls, aircraft landing gear and barrels of gun/cannon tubes etc. due to their high hardness, excellent wear and corrosion resistance, high melting point and low coefficient of friction [1-4]. However, under the severe service conditions such as high temperature, gas erosion and severe stressing, damage is easily caused at the substrate/chromium interface and results in the spallation of the local chromium coatings along the interface [5-7]. With more and more severe operating conditions, the single traditional chromium plating cannot meet the practical requests. In order to improve the damage-resistance ability of the substrate/Cr plating interface,

\footnotetext{
* Corresponding author. Tel.: +86 1062547527 1; fax: +86 1062561284 .

E-mail address: 1hx1022@126.com (H. Li).
}

various duplex surface techniques based on chromium plating, such as the combinations of chromium plating plus lasersurface hardening (or plasma nitriding), were proposed and investigated [8-11]. The duplex techniques of chromium plating and laser-surface hardening include two typical hybrid processes: laser hardening of chromium-plated steel and the duplex process of substrate laser pre-quenching plus chromium post-depositing. The former was ever studied in the late 1970s and its results showed that the durability of the lasertreated chromium plate was largely decreased [8]. The latter has been successfully utilized to greatly improve the service life of the chromium-coated parts [12,13], but its mechanism has not been revealed yet.

It is well known that the microstructure and morphologies of the substrate/coating interface play an important role in the service life of coatings. To discuss the influences of the laser pre-quenching on the morphologies and microstructure of the substrate/Cr interface, the interface of the laserdiscrete-quenched steel substrate and as-deposited chromium coatings was studied by ion beam etching, dissolving- 


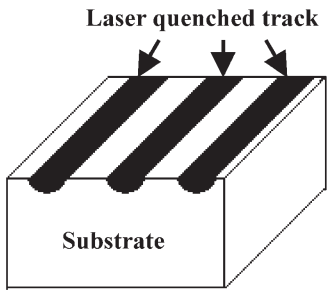

(a) Schematic of laser-quenching

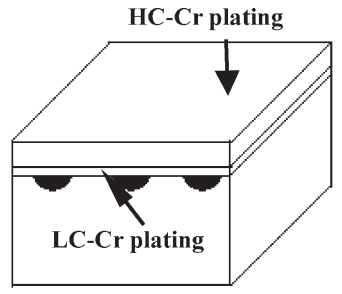

(b) schematic of chromium electroplating

Fig. 1. Processing schematics of laser quenching and chromium electroplating.

substrate-away and using a Vickers hardness tester in the present paper.

\section{Experimental}

\subsection{Preparation of a laser-discrete-quenched substrate and chromium system}

The substrate material was as-quenched and tempered $30 \mathrm{CrNi} 2 \mathrm{MoV}$ (AISI 3034) steel (main chemical ingredients: $0.28 \mathrm{C}, 0.7 \mathrm{Cr}, 2.27 \mathrm{Ni}, 0.20 \mathrm{Mo}, 0.21 \mathrm{~V}$, all in wt.\%). The steel plate $40 \times 40 \times 15 \mathrm{~mm}$ was discretely quenched using a continuous Nd:YAG laser with beam power $150 \mathrm{~W}$, beam diameter $0.5 \mathrm{~mm}$ and beam travel velocity $25 \mathrm{~mm} / \mathrm{s}$. The beam interval between two laser tracks is about $0.5 \mathrm{~mm}$. The quenched steel plate was tempered at $200{ }^{\circ} \mathrm{C}$ for $2 \mathrm{~h}$ to remove stresses. The samples $20 \times 20 \times 15 \mathrm{~mm}$ were cut off using an electro-sparking machine, and their laser-quenched surfaces were mechanically ground and polished. In this paper, the chromium coatings, which were composed of low-contraction (LC) and high-contraction (HC) chromium, were prepared by the commercial electroplating processing of the practical chromium-coated parts. The LC-Cr layer about $20 \mu \mathrm{m}$ thick was pre-deposited as an interlayer with the commercial plating bath of chromic acid $\left(250 \mathrm{~g} \mathrm{l}^{-1}\right)$ and sulfuric acid $\left(2.5 \mathrm{~g} \mathrm{l}^{-1}\right)$, at a temperature of $85{ }^{\circ} \mathrm{C}$ and a current density of $60 \mathrm{~A} / \mathrm{dm}^{2}$. The HC-Cr plate approximately $100 \mu \mathrm{m}$ thick was deposited at a lower bath temperature and a lower current density. The processing schematics of laser quenching and chromium electroplating are shown in Fig. 1a and b, respectively. The plated samples were subjected to vacuum annealing at $200{ }^{\circ} \mathrm{C}$ for $2 \mathrm{~h}$ to dehydrogenate.

\subsection{Preparation of samples}

The metallographic samples for ion etching were prepared according to the method and parameters offered in the reference literatures [14-16]. Samples were cut off using an electrosparking machine, and the cross-sections were mechanically ground and polished. Ion etching was performed by using an ion

Table 1

Ion etching parameters [14]

\begin{tabular}{llll}
\hline Current $/ \mathrm{mA}$ & Voltage $/ \mathrm{V}$ & Time $/ \mathrm{min}$ & Incidence angle $/\left({ }^{\circ}\right)$ \\
\hline 60 & 1000 & 30 & 45 \\
\hline
\end{tabular}

sputtering apparatus (KYKY-FD1500) and the ion gas was argon. The detailed parameters are given in Table 1.

In order to explain the interfacial morphologies of the ionetched specimens and further reveal the characteristics of the substrate/Cr interface, the cross-sectional substrates of the substrate/Cr samples were deep etched by nital $4 \%$ in volume and the near-substrate surfaces of the free-standing chromium coatings were studied. The preparation method of the freestanding chromium coatings is as follows: thin slices $20 \times 4 \times$ $0.5 \mathrm{~mm}$ were first cut off from the chromium-plated samples using an electro-sparking machine. And then their substrates were dissolved away by nital $30 \%$ in volume. Finally, the obtained free-standing chromium coatings were ultrasonically cleaned in alcohol.

\subsection{Methods of investigation}

The morphological observations of the specimens were carried out by using Optical Microscope (OM) and High Resolution Scanning Electron Microscope (HRSEM) (FEI Sirion $400 \mathrm{NC}$ ). The hardness of the substrate and chromium coatings was measured using a MH-6 Vickers hardness tester with loads of $9.81 \mathrm{~N}$ (HV1).

\section{Results and discussion}

\subsection{Low-magnification cross-sectional view}

The cross-sectional characteristics of the laser-quenched substrate/Cr specimens etched by nital $2 \%$ in volume are presented in Fig. 2. The laser-quenched tracks in crescent shape are obvious and marked by arrows. Their unique shape is the

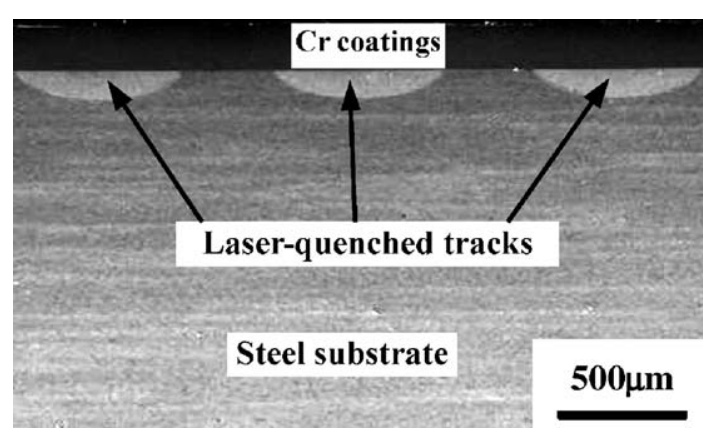

Fig. 2. Cross-sectional view of the laser-discrete-quenched substrate/Cr specimens. 


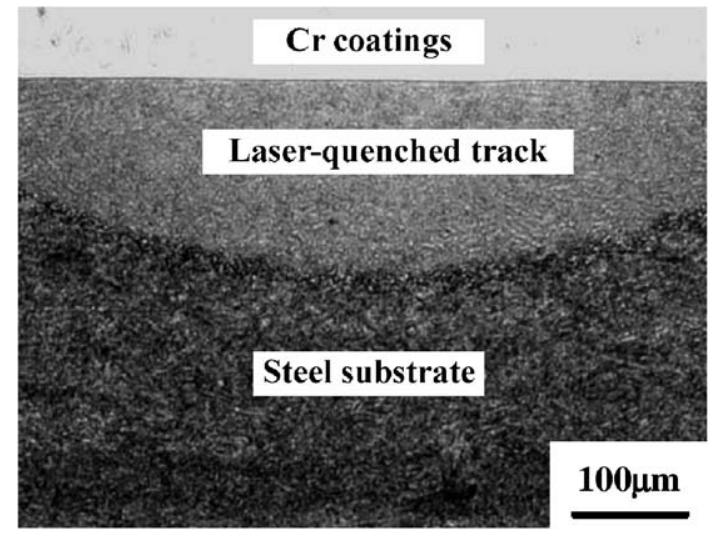

Fig. 3. Typical optical micrograph of the laser-quenched track/Cr cross-section.

result of Gaussian distribution of laser facular energy density. The width and depth of a laser-quenched track are appropriately 600 and $200 \mu \mathrm{m}$, respectively. The spacing between two laser tracks is about $400 \mu \mathrm{m}$. According to Figs. 1 and 2, it is obvious that the periodical microstructure of the substrate surface can be achieved due to the laser-discrete-quenching. As shown in Fig. 3 , the microstructures of the original substrate and laserquenched zone are distinctly different. The microstructure of the original substrate is tempered sorbite while that of the laserquenched zone is very fine. Moreover, the laser-quenched tracks are composed of gradient microstructure.

\subsection{Microhardness measurements}

Fig. 4 shows the substrate hardness gradient over the depth of a laser-quenched-track center. Maximum hardness values of about 650 HV1 were measured in the laser-quenched track, and the average hardness of the original substrate was appropriately $340 \mathrm{HV} 1$. In addition, the average hardness values of the LC-Cr and HC-Cr were about 670 and $960 \mathrm{HV} 1$, respectively. Accordingly, an interfacial interlayer with hardness gradient between the substrate and $\mathrm{Cr}$ plating is achieved by the laser quenching. When the substrate/coating compound has to withstand high loads, poor hardness of the substrate would prevent industrial application of excellent coating properties. Thus, the laser pre-quenching can reduce the steep hardness gradient at the substrate/chromium interface and improve the load-bearing capacity of chromium electrodeposits, which maybe benefits for increasing the service life of the chromium-coated parts.

\subsection{High-magnification cross-sectional micrograph}

Fig. 5 shows the cross-sectional morphologies of the substrate/chromium interface etched by ion beam. The extremely different morphologies of the LC-Cr/original substrate and LC-Cr/laser-quenched center interfaces are presented in Fig. 5a and b, respectively. There is an about $2 \mu \mathrm{m}$ transient interlayer at the former while the latter looks smooth and straight. The EDS analysis proves that the transient interlayer is mainly composed of $\mathrm{Fe}$ and $\mathrm{Cr}$ atoms.
Fig. 6 presents the cross-sectional SEM micrographs of the substrate/Cr specimens whose substrates were deep etched by nital $4 \%$ in volume. The gradient microstructure and morphologies of the laser-quenched track are shown in Fig. 6a. The zone $\mathrm{A}, \mathrm{B}$ and $\mathrm{C}$ in Fig. $6 \mathrm{a}$ are the cross-sections of the laserquenched zone, transition zone and original substrate/LC-Cr interfaces, respectively. The laser-quenched tracks in crescent shape are marked by arrow. Fig. 6b, c and d show the high magnification SEM micrographs taken in zones A through $\mathrm{C}$, respectively. As shown in Fig. 6, it is obvious that the microstructures of the laser-quenched zone, transition zone and original substrate are distinctly different. The laserquenched zone may be composed of lath martensite and extremely fine carbide particles while the original substrate contains many small carbide particles (in Fig. $6 \mathrm{~b}$ and d). The extremely uneven microstructure and morphologies of the transition zone are shown in Fig. 6c. Due to the different microstructures, the above three zones have dissimilar physical and chemical properties. It is well known that the corrosion resistance of the transition zone and original substrate is poorer than that of the laser-quenched zone. When the laser-quenched substrates were pretreated by acid pickling and anodic etching before electroplating, the surfaces of the transition zone and original substrate became rougher. Accordingly, the $\mathrm{LC}-\mathrm{Cr}$ / laser-quenched-zone interface is smoother than that of the other interfaces (Fig. 6).

\subsection{Near-substrate surface characteristics of the free-standing chromium coatings}

Fig. 7 presents the near-substrate surface characteristics of the free-standing Cr coatings. The EDS analysis proves that the chemical composition of the near-substrate surface is predominantly $\mathrm{Cr}$. The low magnification initial morphologies and microstructure of the LC-Cr layer are shown in Fig. 7a. The zone $\mathrm{A}, \mathrm{B}$ and $\mathrm{C}$ in Fig. 7a are corresponding to the lasertransformation hardened zone, transition region and original substrate, respectively. As shown in Figs. 6 and 7, it is obvious

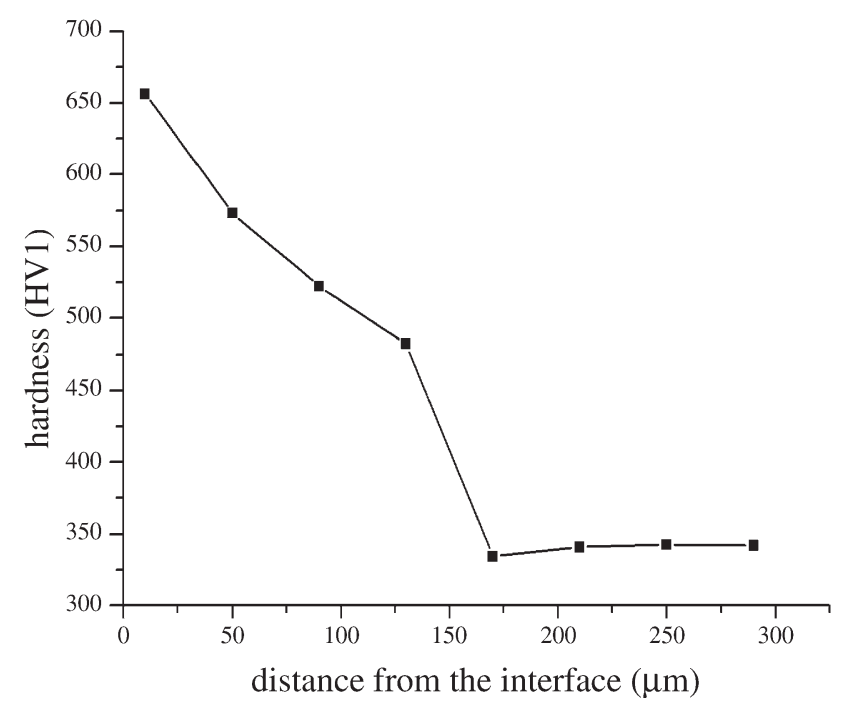

Fig. 4. Substrate hardness over the depth of the laser-quenched-track center. 

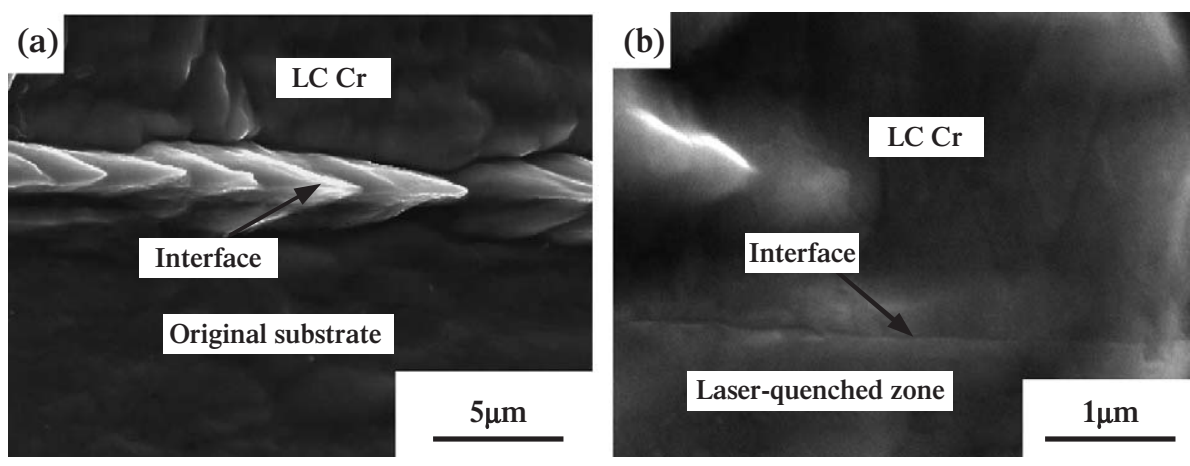

Fig. 5. SEM micrographs of the LC-Cr/substrate interfacial morphologies etched by ion beam; (a) interfacial morphologies of LC-Cr and original substrate; (b) interfacial morphology of LC-Cr and laser-quenched zone.

that the initial morphologies and microstructure of the $\mathrm{LC}-\mathrm{Cr}$ layer show the same periodic characteristics as those of the laser-quenched substrate surface. The typical HRSEM micrographs taken in zones A through $\mathrm{C}$ are presented in Fig. 7a, b and $\mathrm{c}$, respectively. It can be observed that the microstructure and morphologies of zones A through C in Fig. 7a are extremely different. The crystal grains of zone A in Fig. 7a are far smaller than those of zones B and C, and they are statistically about 7$12 \mathrm{~nm}$ in diameter. The refinement of the crystal grains in zone A may be caused by a large number of dislocations and other defects in the substrate laser-quenched zone, for these dislocations and defects can offer more nuclei for electrodeposition of $\mathrm{Cr}$ atoms. Fig. $7 \mathrm{~d}$ shows a typical micro-dimple in zone $C$ with the crystal grains of statistically $30-40 \mathrm{~nm}$ in diameter. The crystal grains (and/or clusters) in zone B are uneven in sizes (Fig. 7c). In addition, the surface of zone A is smoother than that of zones B and C. Due to the identical electroplating parameters, the different microstructures of the laser-quenched zone, transition zone and original substrate are responsible for the distinct difference of zones A through $\mathrm{C}$. Thus, it can be well concluded that the laser-quenched microstructure has a great effect on the growth patterns and properties of the initial LC-Cr layer.

\subsection{Discussion}

Based on the above results, the laser quenching prior to plating has the following effects on the substrate/Cr interface: changing the microstructure and properties of the substrate surface, reducing the steep hardness gradient at the substrate/
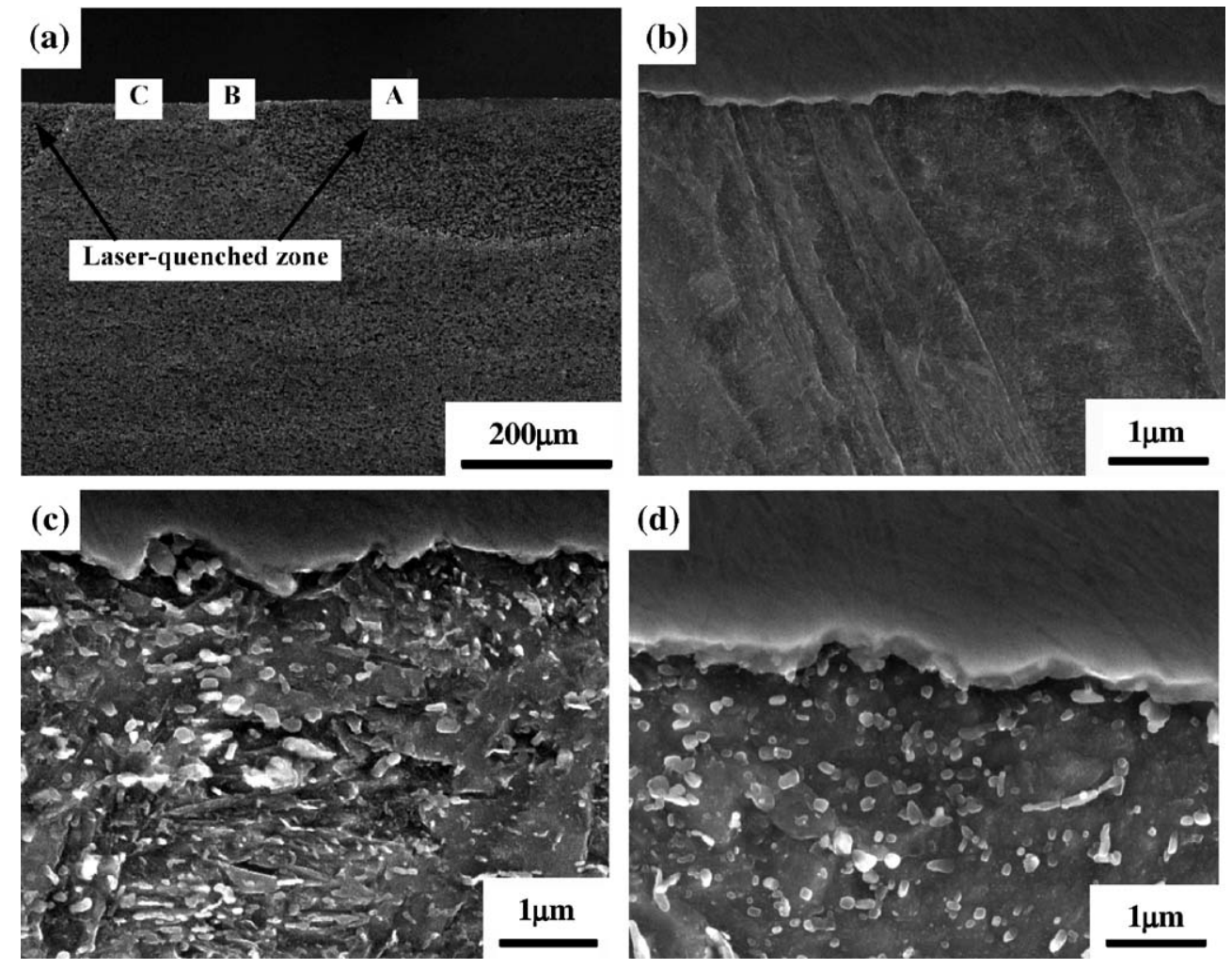

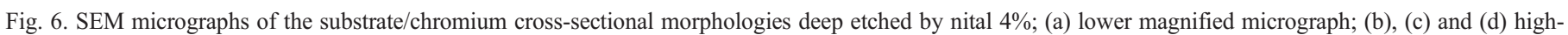
magnified micrographs of the $\mathrm{A}, \mathrm{B}$ and $\mathrm{C}$ zones in (a), respectively. 

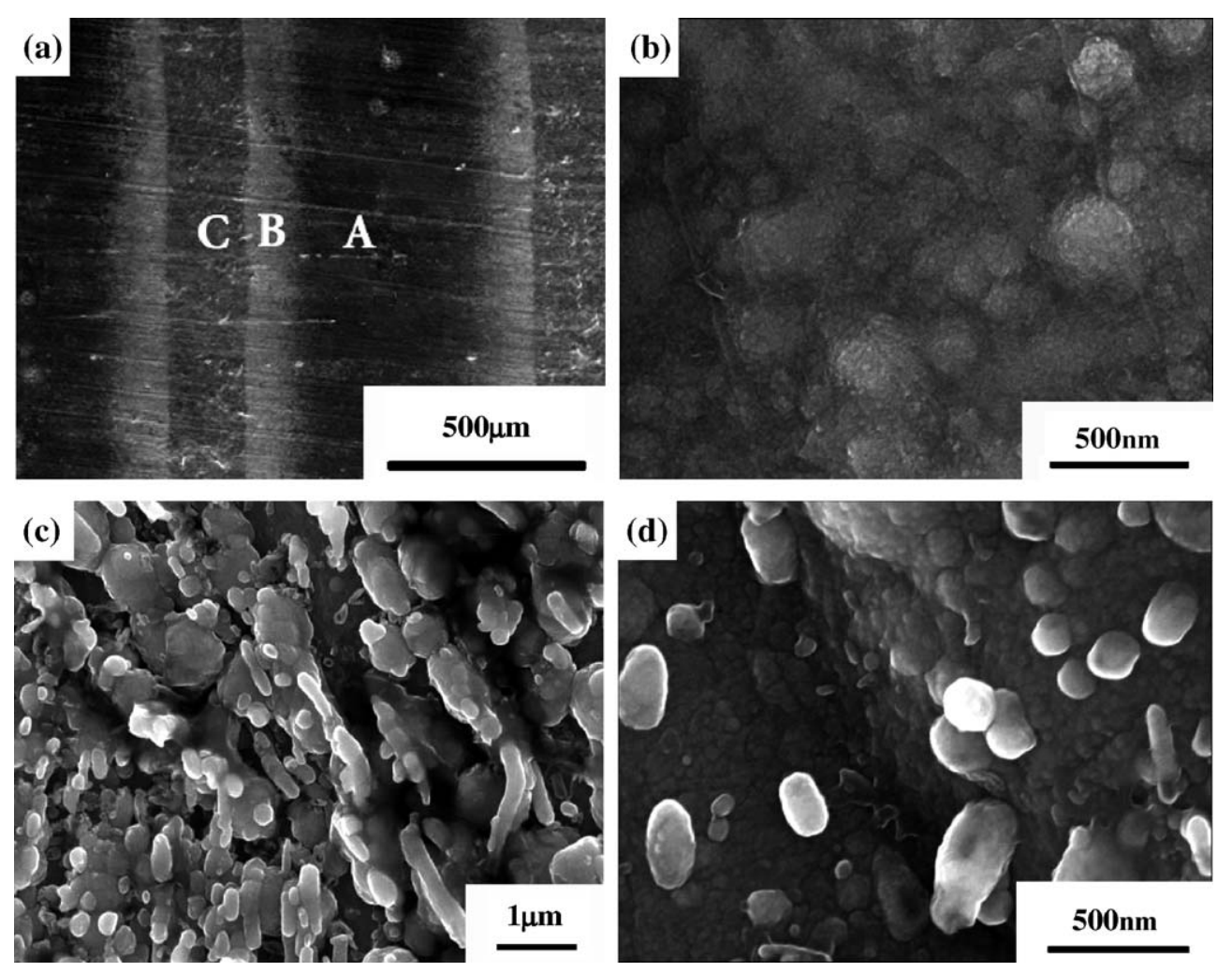

Fig. 7. SEM micrographs of the typical near-substrate surface characteristics of the free-standing Cr electrodeposits; (a) low-magnified micrograph, the three zones of distinctly different morphologies are labeled zones A, B and C; (b), (c) and (d) HRSEM micrographs corresponding to zones A, B and C in (a), respectively.

chromium interface, improving the load-bearing capacity of chromium electrodeposits and altering the initial microstructure and morphologies of the LC-Cr layer. As shown in Figs. 2 and 6 , the periodically gradient microstructure of the substrate surface is fabricated after laser quenching. Due to the different microstructure and corrosion-resistance of the laser-quenched zone, transition zone and original substrate, the periodically gradient morphologies of the substrate surface can be produced by the pretreatment processes (such as acid pickling and anodic etching) prior to electroplating. Moreover, it is surprising that the initial microstructure and morphologies of the LC-Cr layer inherit the periodically gradient characteristics of the substrate surface (Fig. 7). The microstructural changes of the substrate and LC-Cr near the laser-quenched track/LC-Cr interface may be responsible for the different interfacial morphologies in Fig. 5. Consequently, the interfacial interlayer with periodically gradient microstructure and properties is generated between the steel substrate and chromium plating. Under the severe service conditions such as high temperature and severe stressing, the graded interfacial layer can effectively reduce the mismatch stress near the substrate/Cr plating interface. Thus, these gradient morphologies and microstructure near the substrate/ $\mathrm{Cr}$ interface may be responsible for improving the service life of the chromium-coated parts in the severe operating conditions.

\section{Conclusions}

There is a transient interlayer at the original substrate/LC-Cr interface while the laser-quenched-zone/LC-Cr interface hasn't the so-called interlayer. This phenomenon is caused by the microstructural changes of the substrate and LC-Cr near the laser-quenched track/LC-Cr interface.

The laser pre-quenching not only changes the microstructure and properties of the laser-quenched regions and improves the load-bearing capacity of electrodeposited chromium coatings, but greatly affects the initial microstructure and morphologies of $\mathrm{Cr}$ electrodeposits, namely, the initial microstructure and morphologies of $\mathrm{Cr}$ electrodeposits can inherit the periodically gradient characteristics of the laser-quenched substrate surface; the interfacial layer with gradient microstructure and properties between the substrate and LC-Cr plating can be formed by the duplex technique of laser pre-quenching plus chromium postdepositing.

\section{Acknowledgments}

The financial support provided by the National Natural Science Foundation of China (Grant No. 50471087 and 50531060) is greatly acknowledged.

\section{References}

[1] J.M. Tyler, Met. Finish. 93 (10) (1995) 10.

[2] K.M. Yin, C.M. Wang, Surf. Coat. Technol. 114 (1999) 213.

[3] J. Simão, D.K. Aspinwall, J. Mater. Process. Technol. 92-93 (1999) 218.

[4] L. Fedrizzi, S. Rossi, F. Bellei, F. Deflorian, Wear 253 (2002) 1173.

[5] B. Lawton, Wear 251 (2001) 827.

[6] S. Sopok, C. Richard, S. Dunn, Wear 258 (2005) 659. 
[7] P.J. Cote, G. Kendall, M.E. Todaro, Surf. Coat. Technol. 146-147 (2001) 65

[8] R.S. Montgomery, Wear 155-166 (1979) 56.

[9] L. Wang, D.S. Kim, K.S. Nam, M. Kim, S.C. Kwon, Surf. Coat. Technol. 151-154 (2005) 190.

[10] L. Wang, K.S. Nam, S.C. Kwon, Appl. Surf. Sci. 372-377 (2003) 207.

[11] E. Menthe, K.-T. Rie, Surf. Coat. Technol. 217-220 (1999) 112.

[12] W. Harald, S. Uvc, Germany. Patent: DE19918794.
[13] G.N. Chen, G.X. Luo, K. Zhang, X.Y. Xu, H. Shen, H.M. Yan, D. Rao, Acta Armament. II(Suppl.) 24 (2003) 6 (in Chinese).

[14] X.Y. Xu, K. Zhang, J.M. Han, G.N. Chen, Trans. Nonferr. Met. Soc. China 14 (2004) 282.

[15] M. Pohl, W.G. Burchard, Scanning 3 (1980) 251.

[16] K.A. Pischow, A.S. Korhonen, M. Adamik, P.B. Barna, Surf. Coat. Technol. 67 (1994) 95. 